\title{
Multi-objective automated guided vehicle scheduling based on MapReduce framework
}

\author{
Shi, W. ${ }^{a, b}$, Tang, D.B. ${ }^{a,}{ }^{,}$, Zou, P. ${ }^{b}$ \\ ${ }^{a}$ College of Mechanical and Electrical Engineering, Nanjing University of Aeronautics and Astronautics, Nanjing, P.R. China \\ ${ }^{b}$ Beijing Aerospace Smart Manufacturing Technology of Development Co., Ltd., Beijing, P.R. China
}

\begin{abstract}
A B S T R A C T
During material handling processes, automated guided vehicles (AGVs) pose a path conflict problem. To solve this problem, we proposed a multi-objective scheduling model based on total driving distance and waiting time, and used the A* path planning algorithm to search the shortest path of AGV. By using a speed control strategy, we were able to detect the overlap path and the conflict time. Additionally, we adopted an efficient MapReduce framework to improve the speed control strategy execution efficiency. At last, a material handling system of smart electrical connectors workshop was discussed to verify the scheduling model and the speed control strategy combined with the MapReduce framework is feasible and effective to reduce the AGV path conflict probability. The material handling system could be applied in workshop to replace manual handling and to improve production efficiency.
\end{abstract}

\author{
ARTICLE INFO \\ Keywords: \\ Automated-guided vehicle(AGV); \\ Scheduling; \\ AGV scheduling; \\ MapReduce; \\ Path planning; \\ $\mathrm{A}^{*}$ search algorithm \\ *Corresponding author: \\ d.tang@nuaa.edu.cn \\ (Tang, D.B.) \\ Article history: \\ Received 25 January 2021 \\ Revised 2 March 2021 \\ Accepted 8 March 2021
}

\section{References}

[1] Fazlollahtabar, H., Saidi-Mehrabad, M. (2015). Methodologies to optimize automated guided vehicle scheduling and routing problems: A review study, Journal of Intelligent \& Robotic Systems, Vol. 77, No. 3-4, 525-545, doi: 10.1007/s10846-013-0003-8.

[2] Sun, Q., Liu, H., Yang, Q., Yan, W. (2011). On the design for AGVs: Modeling, path planning and localization, In: Proceedings of the 2011 IEEE International Conference on Mechatronics and Automation, Beijing, China, 15151520, doi: 10.1109/ICMA.2011.5985974.

[3] Yershov, S., LaValle, S.M. (2012). Simplicial Dijkstra and A* algorithms: From graphs to continuous spaces, $\mathrm{Ad-}$ vanced Robotics, Vol. 26, No. 17, 2065-2085, doi: 10.1080/01691864.2012.729559.

[4] Wang, C.L., Li, S.W. (2018). Hybrid fruit fly optimization algorithm for solving multi-compartment vehicle routing problem in intelligent logistics, Advances in Production Engineering \& Management, Vol. 13, No. 4, 466-478, doi: 10.14743/apem2018.4.304.

[5] Wang, C., Wang, L., Qin, J., Wu, Z., Duan, L., Li, Z., Cao, M., Ou, X., Su, X., Li, W., Lu, Z., Li, M., Wang, Y., Long, J., Huang, M., Li, Y., Wang, Q. (2015). Path planning of automated guided vehicles based on improved A-star algorithm, In: Proceeding of the 2015 IEEE International Conference on Information and Automation, Lijiang, China, 2071-2076, doi: 10.1109/ICInfA.2015.7279630.

[6] Lau, H.Y.K., Zhao, Y. (2008). Integrated scheduling of handling equipment at automated container terminals, Annals of Operations Research, Vol. 159, 373-394, doi: 10.1007/s10479-007-0262-5. 
[7] Liu, Y., Ji, S., Su, Z., Guo, D. (2019). Multi-objective AGV scheduling in an automatic sorting system of an unmanned (intelligent) warehouse by using two adaptive genetic algorithms and a multi-adaptive genetic algorithm, PLOS ONE, Vol. 14, No. 12, Article ID e0226161, doi: 10.1371/journal.pone.0226161.

[8] Li, G., Zeng, B., Liao, W., Li, X., Gao, L.(2018). A new AGV scheduling algorithm based on harmony search for material transfer in a real-world manufacturing system, Advances in Mechanical Engineering, Vol. 10, No, 3, Article No. 1687814018765560, doi: 10.1177/1687814018765560.

[9] Yang, M.S., Ba, L., Zheng, H.Y., Liu, Y., Wang, X.F., He, J.Z., Li, Y. (2019). An integrated system for scheduling of processing and assembly operations with fuzzy operation time and fuzzy delivery time, Advances in Production Engineering \& Management, Vol. 14, No. 3, 367-378, doi: 10.14743/apem2019.3.334.

[10] Zhao, X.F., Liu, H.Z., Lin, S.X., Chen, Y.K. (2020). Design and implementation of a multiple AGV scheduling algorithm for a job-shop, International Journal of Simulation Modelling, Vol. 19, No. 1, 134-145, doi: 10.2507/ IJSIMM19-1-CO2.

[11] Wang, J.F., Liu, J.H., Zhong, Y.F. (2005). A novel ant colony algorithm for assembly sequence planning, The International Journal of Advanced Manufacturing Technology, Vol. 25, 1137-1143, doi: 10.1007/s00170-003-1952-z.

[12] Reed, M., Yiannakou, A., Evering, R. (2014). An ant colony algorithm for the multi-compartment vehicle routing problem, Applied Soft Computing, Vol. 15, 169-176, doi: 10.1016/i.asoc.2013.10.017.

[13] Balseiro, S.R., Loiseau, I., Ramonet, J. (2011). An ant colony algorithm hybridized with insertion heuristics for the time dependent vehicle routing problem with time windows, Computers \& Operations Research, Vol. 38, No. 6, 954-966, doi: 10.1016/j.cor.2010.10.011.

[14] Mousavi, M., Yap, H.J., Musa, S.N., Dawal, S.Z.M. (2017). A fuzzy hybrid GA-PSO algorithm for multi-objective AGV scheduling in FMS, International Journal of Simulation Modelling, Vol. 16, No. 1, 58-71, doi: 10.2507/IJSIMM16 (1) 5.368.

[15] Sadeghpour, H., Tavakoli, A., Kazemi, M., Pooya, A. (2019). A novel approximate dynamic programming approach for constrained equipment replacement problems: A case study, Advances in Production Engineering \& Management, Vol. 14, No. 3, 355-366, doi: 10.14743/apem2019.3.333.

[16] Xu, W., Yin, Y. (2018). Functional objectives decision-making of discrete manufacturing system based on integrated ant colony optimization and particle swarm optimization approach, Advances in Production Engineering \& Management, Vol. 13, No. 4, 389-404, doi: 10.14743/apem2018.4.298.

[17] Li, H.-Y., Xu, W., Cui, Y., Wang, Z., Xiao, M., Sun, Z.-X. ( 2020). Preventive maintenance decision model of urban transportation system equipment based on multi-control units, IEEE Access, Vol. 8, No. 1, 15851-15869, doi: 10.1109/ACCESS.2019.2961433.

[18] Liu, S.N., Ke, Y.L. (2007). An algorithm for job shop scheduling in dual resource constrained with AGV, China Mechanical Engineering, Vol. 15, 1810-1813.

[19] Li, X. (2012). Utilization control optimization of AGV based on queuing theory, Journal of Lanzhou Jiaotong University, Vol. 31, No. 6, 91-93.

[20] Jiang, C., Xi, J.T. (2019). Dynamic scheduling in the engineer-to-order (ETO) assembly process by the combined immune algorithm and simulated annealing method, Advances in Production Engineering \& Management, Vol. 14, No. 3, 271-283, doi: 10.14743/apem2019.3.327.

[21] Zheng, K., Tang, D., Giret, A., Gu, W., Wu, X. (2015). Dynamic shop floor re-scheduling approach inspired by a neuroendocrine regulation mechanism, Proceedings of the Institution of Mechanical Engineers, Part B: Journal of Engineering Manufacture, Vol. 229, No, 1, 121-134, doi: 10.1177/0954405414558699.

[22] Zheng, K., Tang, D., Gu, W., Dai, M. (2013). Distributed control of multi-AGV system based on regional control model, Production Engineering, Research and Development, Vol. 7, No. 4, 433-441, doi: 10.1007/s11740-0130456-4.

[23] Rong, J. (2017). Research on collision avoidance strategy of AGV, In: Proceedings of 2017 International Conference on Manufacturing Construction and Energy Engineering (MCEE 2017), Hong Kong, 15754-25995, doi: 10.12783/ dtetr/mcee2017/15754.

[24] Dean, J., Ghemawat, S. (2008). MapReduce: Simplified data processing on large clusters, Communications of the ACM, Vol. 51, No. 1, 107-113, doi: 10.1145/1327452.1327492.

[25] Eken, S., Sayar, A. (2019). A MapReduce-based big spatial data framework for solving the problem of covering a polygon with orthogonal rectangles, Tehnički Vjesnik - Technical Gazette, Vol. 26, No. 1, 36-42, doi: 10.17559/ TV-20170418094421.

[26] Talbot, J., Yoo, R.M., Kozyrakis, C. (2011). Phoenix++: Modular mapreduce for shared-memory systems, In: Proceedings of the Second International Workshop on MapReduce and Its Applications, New York, USA, 9-16, doi: $\underline{10.1145 / 1996092.1996095 .}$ 


\section{APEM}

\title{
Večkriterijsko terminiranje samodejno vodenih vozil, ki temelji na softverskem okolju MapReduce
}

\author{
Shi, W..$^{a, b}$, Tang, D.B. ${ }^{a,}{ }^{*}$, Zou, P. ${ }^{b}$
}

aCollege of Mechanical and Electrical Engineering, Nanjing University of Aeronautics and Astronautics, Nanjing, P.R. China bBeijing Aerospace Smart Manufacturing Technology of Development Co., Ltd., Beijing, P.R. China

\section{POVZETEK}

Pri transportu materiala se ob uporabi samodejno vodenih vozil (AGV) pojavi težava nastanka spora na poti. Za rešitev te težave smo vzpostavili model večkriterijskega razporejanja, ki temelji na skupni vozni razdalji in čakalnem času, ter za iskanje najkrajše poti AGV uporabili algoritem $\mathrm{A}^{*}$. Z uporabo strategije nadzora hitrosti smo zaznali prekrivanje poti in časa. Za izboljšanje učinkovitosti izvajanja strategije nadzora hitrosti smo uporabili softversko okolje MapReduce. Model smo preizkusili na sistemu za transport materiala v delavnici pametnih električnih konektorjev. Cilj je bil preveriti, ali je model razporejanja s strategijo nadzora hitrosti v kombinaciji z okoljem MapReduce izvedljiv in učinkovit za zmanjšanje verjetnosti nastanka konfliktnih poti samodejno vodenih vozil. Sistem za transport materiala bi lahko uporabili v delavnici, da bi nadomestili ročni transport material in izboljšali učinkovitost proizvodnje.

\author{
PODATKI O ČLANKU \\ Ključne besede: \\ Samodejno vodeno vozilo (AGV); \\ Razporejanje; \\ Razporejanje AGV; \\ MapReduce; \\ Načrtovanje poti; \\ $A^{*}$ iskalni algoritem \\ *Kontaktna oseba: \\ d.tang@nuaa.edu.cn \\ (Tang, D.B.) \\ Zgodovina članka: \\ Prejet 25. januarja 2021 \\ Popravljen 2. marca 2021 \\ Sprejet 8. marca 2021

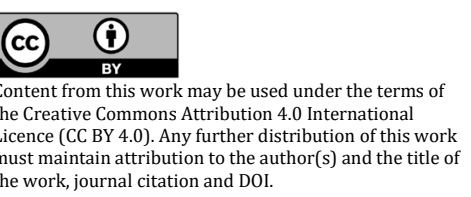

